\title{
The Ellipsometrical Analysis of External Reflection of Light on Superficial Films on Solid Substrates
}

\author{
Simion Jitian \\ Politehnica University Timisoara, Faculty of Engineering, Hunedoara, Romania
}

Email address:

jitian_s@yahoo.com (S. Jitian)

To cite this article:

Simion Jitian. The Ellipsometrical Analysis of External Reflection of Light on Superficial Films on Solid Substrates. American Journal of Optics and Photonics. Vol. 3, No. 4, 2015, pp. 48-53. doi: 10.11648/j.ajop.20150304.12

\begin{abstract}
The ellipsometrical analysis of the external specular reflection of light on nonabsorbing superficial films allows us to know factors which influence the ellipsometric measurement of the analyzed system. For optical nonabsorbing superficial films the curves $\Delta=\mathrm{f}\left(\mathrm{d}_{\mathrm{f}}\right)$ and $\Psi=\mathrm{f}\left(\mathrm{d}_{\mathrm{f}}\right)$ are periodical, while the curves $\Delta=\mathrm{f}(\Psi)$ are closed. The paper presents observations on the dependence of the ellipsometric parameters $\Delta$ and $\Psi$ on $\mathrm{d}_{\mathrm{f}}$. The analysis of the periodicity of these curves allows us to correctly determine the film thickness for thicknesses greater than $d_{\min }$. The value of $d_{\min }$ depends on the refractive index $n_{f}$ of the surface film, the incidence angle $\varphi_{0}$ and the wavelength $\lambda$ of the incident radiation. The dependence of $d_{\min }$ on $n_{f}, \varphi_{0}$ and $\lambda$ is analyzed. From the curve shape we can draw conclusions with respect to the domain of small errors, allowing us to correctly determine the thickness and refraction index of superficial films.
\end{abstract}

Keywords: Ellipsometry, Superficial Film, External Specular Reflection

\section{Introduction}

In the case of reflection of monochromatic light radiation on the surface of a homogeneous and isotropic, optically non-absorbent surface film deposited on a solid support reflector, there is a change of the polarization state of light. Figure 1 is the schematic model of the external reflection of monochromatic radiation on an optically absorbent surface film.

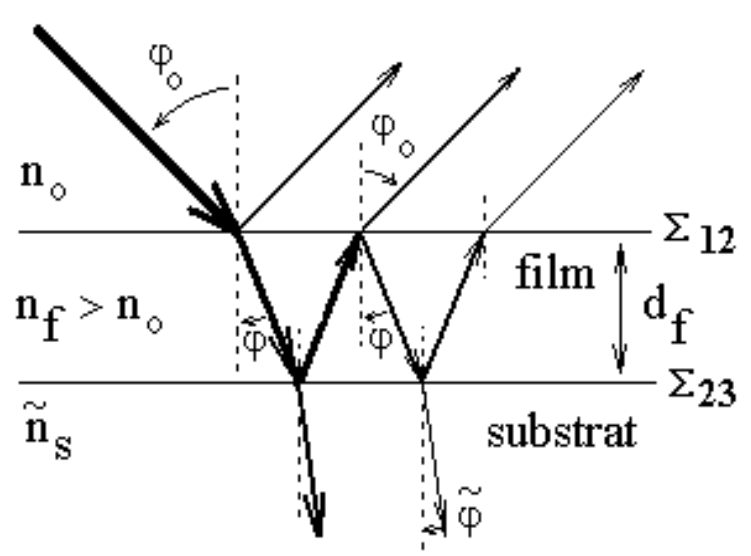

Figure 1. The external reflection of a monochromatic plane polarized radiation on the substrate-film surface.
The modification of the polarization state of the reflected radiation can be ellipsometrically measured [1]. $\Delta$ and $\Psi$ are the parameters that can be measured. The $\Delta$ parameter expresses the phase angle between the light components, parallel to the plane of incidence and perpendicular to the plane of incidence, after reflection. Here, $\tan \Psi$ is the relative attenuation of two components, after reflection.

The complex parameter $\tilde{\rho}$ can be calculated depending on the parameters $\Delta$ and $\Psi$ by the relation:

$$
\begin{aligned}
& \widetilde{\rho}=\tan \Psi \cdot \exp (i \Delta)=\tan \Psi \cos \Delta+\mathrm{i} \tan \Psi \sin \Delta= \\
& =\operatorname{Re} \widetilde{\rho}+\mathrm{i} \operatorname{Im} \widetilde{\rho}=\frac{\widetilde{\mathrm{R}}_{\mathrm{p}}}{\widetilde{\mathrm{R}}_{\mathrm{s}}}
\end{aligned}
$$

by taking into account the reflections and therefore the attenuation and phase shift on all interfaces.

The complex parameters $\widetilde{\mathrm{R}}_{\mathrm{p}}$ and $\widetilde{\mathrm{R}}_{\mathrm{s}}$ are expressed in terms of complex Fresnel reflection coefficients corresponding to reflections on the two interfaces $\Sigma_{12}$ and $\Sigma_{23}$ : $\widetilde{\mathrm{r}}_{\mathrm{p}}^{12}, \widetilde{\mathrm{r}}_{\mathrm{p}}^{23}, \widetilde{\mathrm{r}}_{\mathrm{s}}^{12}, \widetilde{\mathrm{r}}_{\mathrm{s}}^{23}[2]:$

$$
\widetilde{\mathrm{R}}_{\mathrm{p}}=\frac{\widetilde{\mathrm{r}}_{\mathrm{p}}^{12}+\widetilde{\mathrm{r}}_{\mathrm{p}}^{23} \exp \widetilde{\mathrm{D}}}{1+\widetilde{\mathrm{r}}_{\mathrm{p}}^{12} \cdot \widetilde{\mathrm{r}}_{\mathrm{p}}^{23} \exp \widetilde{\mathrm{D}}} \text { and } \widetilde{\mathrm{R}}_{\mathrm{s}}=\frac{\widetilde{\mathrm{r}}_{\mathrm{s}}^{12}+\widetilde{\mathrm{r}}_{\mathrm{s}}^{23} \exp \widetilde{\mathrm{D}}}{1+\widetilde{\mathrm{r}}_{\mathrm{s}}^{12} \cdot \widetilde{\mathrm{r}}_{\mathrm{s}}^{23} \operatorname{exp~} \widetilde{\mathrm{D}}}
$$

where: 


$$
\widetilde{D}=-\frac{4 \pi i d_{f}}{\lambda} \sqrt{n_{f}^{2}-n_{0}^{2} \sin ^{2} \varphi_{0}}
$$

The expression: exp $\widetilde{\mathrm{D}}$ is a regular function that depends on the thickness and refractive index of the film surface. Also, that expression depends on incidence angle and wavelength of the incidence radiation.

\section{The Dependence of the Ellipsometrical Parameters $\Delta$ and $\Psi$ on Superficial Film Thickness}

In the case of external reflection, $\mathrm{n}_{\mathrm{f}}>\mathrm{n}_{0}$. The complex parameters: $\widetilde{R}_{p}, \widetilde{R}_{s}$ and $\widetilde{\rho}$ should be repeated after a particular value of the film thickness $d_{f}$, for which $\widetilde{D}$ is a multiple of $2 \pi \mathrm{i}$.

The ellipsometrical readings $\Delta$ and $\Psi$, calculated from relations:

$$
\Delta=\operatorname{arctg} \frac{\operatorname{Im} \tilde{\rho}}{\operatorname{Re} \tilde{\rho}}, \Psi=\operatorname{arct} \Phi \tilde{\rho}
$$

are also periodical functions of the superficial film thickness, having the same period. For this reason, the curves $\Delta=\mathrm{f}(\Psi)$, shown in Figure 2, are closed loops [3].

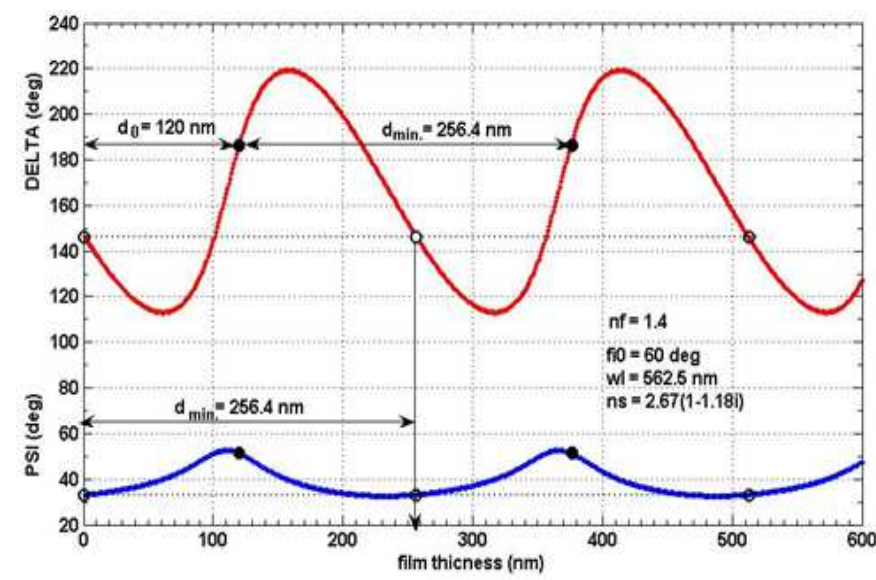

a)

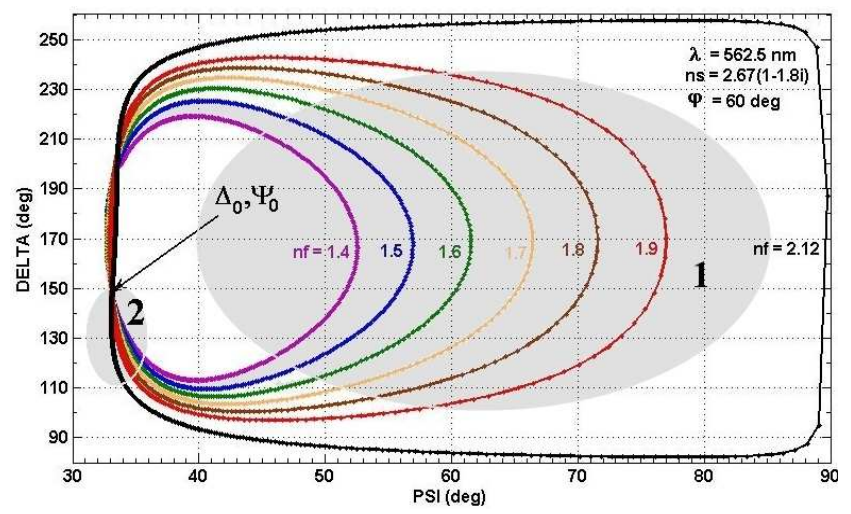

Figure 2. The curves $\Delta=f(\Psi)$ for superficial films with $n_{f}=1.2 \div 2.5$, deposited on a metallic substrate. $n_{s}=2.67(1-1.18 i) ; \varphi_{0}=60^{\circ} ; \lambda=562.5 \mathrm{~nm}$. $\Delta_{0}$ and $\Psi_{0}$ are the ellipsometric measured parameters for the substrate free of film.

Figure 3 shows the variation of the ellipsometrical readings $\Delta$ and $\Psi$ according to the superficial film thickness.

The minimum of film thickness for which the exponential function $\exp \widetilde{D}$ is repeated is $\mathrm{d}_{\min }$.

$$
\mathrm{d}_{\mathrm{f}}=\mathrm{m} \frac{\lambda}{2 \sqrt{\mathrm{n}_{\mathrm{f}}^{2}-\mathrm{n}_{0}^{2} \sin ^{2} \varphi_{0}}}=\mathrm{m} \cdot \mathrm{d}_{\min } ; \mathrm{m}=1,2,3, \ldots
$$

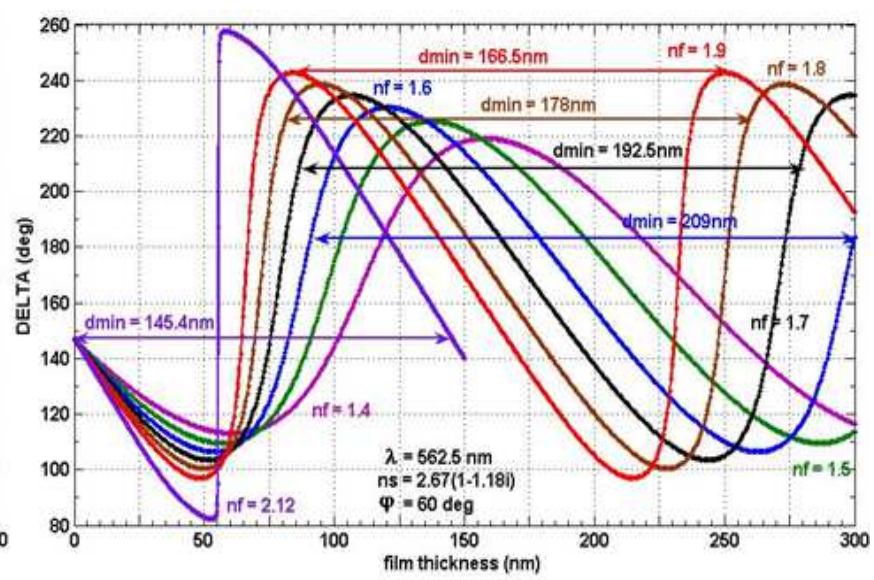

b)

Figure 3. The curves $\Delta=f\left(d_{f}\right)$ and $\Psi=f\left(d_{f}\right)$ for superficial films, deposited on a metallic substrate. $n_{s}=2.67(1-1.18 i) ; \varphi_{0}=60^{0} ; \lambda=562.5 \mathrm{~nm}$.

The film thickness $d_{f}$ is real because $n_{f}>n_{0}$. The minimum film thickness for which values of $\Delta$ and $\Psi$ is repeated is $\mathrm{d}_{\min }$.

You can determine the exact thickness of an optical nonabsorbent film, by ellipsometrical measurements, only if it is thinner than $d_{\text {min }}$. For thicker films, the thickness shall be expressed by the relationship:

$$
\mathrm{d}_{\mathrm{f}}=\mathrm{d}_{0}+\mathrm{m} \cdot \mathrm{d}_{\min }
$$

where $\mathrm{d}_{0}$ is the thickness, determined by ellipsometrical measurement, and $\mathrm{m}$ is an integer. For example, for $\Delta=186.37^{\circ}$ and $\Psi=51.317^{\circ}$, the film thickness with $\mathrm{n}_{\mathrm{f}}=1.4$ can be $120 \mathrm{~nm}$ or $\mathrm{d}_{\mathrm{f}}=(120+\mathrm{m} \cdot 256.4) \mathrm{nm} ; \mathrm{m}=1,2,3, \ldots$ as shown in Figure $3 \mathrm{a}$.
For films with thicknesses greater than $d_{\text {min }}$, further measurements are needed to determine the thickness, due to the periodicity of the functions $\Delta=\mathrm{f}\left(\mathrm{d}_{\mathrm{f}}\right)$ and $\Psi=\mathrm{f}\left(\mathrm{d}_{\mathrm{f}}\right)$. Usually, interferometer measurements are used to determine the order of magnitude of $\mathrm{d}_{\mathrm{f}}$, with a precision of $0.1 \mu \mathrm{m}[4,5,6]$. Typically, the thickness $d_{\min }$ value is on the order of $0.3 \div 0.5 \mu \mathrm{m}$. It is then necessary to determine the exact thickness, by an ellipsometrical measurement, with an accuracy of $0.1 \mathrm{~nm}$.

The value of $d_{\min }$ depends on the refractive index $n_{f}$ of the surface film as well as on the measuring conditions: the incidence angle $\varphi_{0}$ and the wavelength $\lambda$ of the incident radiation, according to the relation (5). 


\subsection{The Dependence of $d_{\min }$ on the Refractive Index $n_{f}$}

Table 1. The values of minimum thickness $d_{\min }$ of the surface film (expressed in $\mathrm{nm}$ ) for which the ellipsometrical parameters $\Delta$ and $\Psi$ is repeated.

\begin{tabular}{rlllllll}
\hline $\boldsymbol{\lambda}$ & $\boldsymbol{\varphi}_{\mathbf{0}}$ & \multicolumn{6}{c}{$\mathbf{n}_{\mathbf{f}}$} \\
\hline $\mathbf{n m}$ & $\mathbf{d e g}$ & $\mathbf{1 . 4}$ & $\mathbf{1 . 6}$ & $\mathbf{1 . 8}$ & $\mathbf{2 . 0}$ & $\mathbf{2 . 1 2}$ & $\mathbf{2 . 2}$ \\
\hline & 50 & 240.0 & 200.2 & 172.7 & 152.2 & 142.3 & 136.4 \\
& 60 & 255.7 & 209.1 & 178.2 & 156.0 & 145.3 & 139.1 \\
562.5 & 70 & 271.0 & 217.2 & 183.2 & 159.3 & 148.0 & 141.4 \\
& 75 & 277.5 & 220.5 & 185.2 & 160.6 & 149.0 & 142.3 \\
& 80 & 282.6 & 223.0 & 186.7 & 161.6 & 149.8 & 143.0 \\
\hline
\end{tabular}

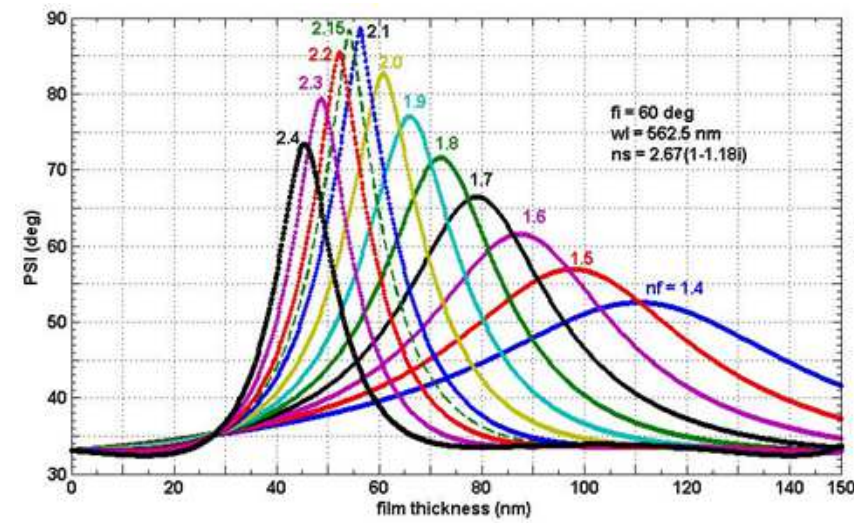

When increasing the refractive index $\mathrm{n}_{\mathrm{f}}$ of the superficial film the value of $d_{\min }$ decreases, as shown in Figure $3 b$ and in Table 1.

The curves $\Psi=\mathrm{f}\left(\mathrm{d}_{\mathrm{f}}\right)$ reach a peak $\left(\Psi=90^{\circ}\right)$ for a particular value of the refractive index of the film and for a particular value of its thickness, as shown in Figure 4.

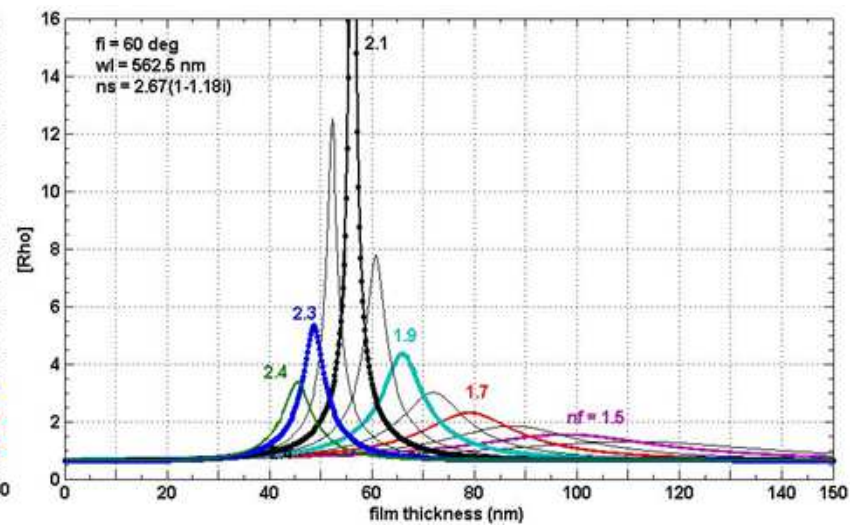

Figure 4. The curves $\Psi=f\left(d_{f}\right)$ and $\mid \tilde{\mathfrak{\rho}}=f\left(d_{f}\right)$ for superficial films, deposited on a metallic substrate. $n_{s}=2.67(1-1.18 i) ; \varphi_{0}=60^{\circ} ; \lambda=562.5 \mathrm{~nm}$.

Then,

$$
\rho=|\widetilde{\rho}|=\frac{\widetilde{R}_{p}}{\widetilde{R}_{s}}=\left|\frac{\frac{\widetilde{r}_{p}^{12}+\widetilde{r}_{p}^{23} \exp \widetilde{D}}{1+\widetilde{r}_{p}^{12} \cdot \widetilde{r}_{p}^{23} \exp \widetilde{D}}}{\frac{\widetilde{r}_{s}^{12}+\widetilde{r}_{s}^{23} \exp \widetilde{D}}{1+\widetilde{r}_{s}^{12} \cdot \widetilde{r}_{s}^{23} \exp \widetilde{D}}}\right| \rightarrow \infty
$$

or

$$
R_{\mathrm{s}}=\left|\widetilde{R}_{\mathrm{s}}\right|=\left|\frac{\widetilde{\mathrm{s}}_{\mathrm{s}}^{12}+\widetilde{\mathrm{r}}_{\mathrm{s}}^{23} \exp \widetilde{\mathrm{D}}}{1+\widetilde{\mathrm{r}}_{\mathrm{s}}^{12} \cdot \widetilde{\mathrm{r}}_{\mathrm{s}}^{23} \exp \widetilde{\mathrm{D}}}\right| \rightarrow 0
$$

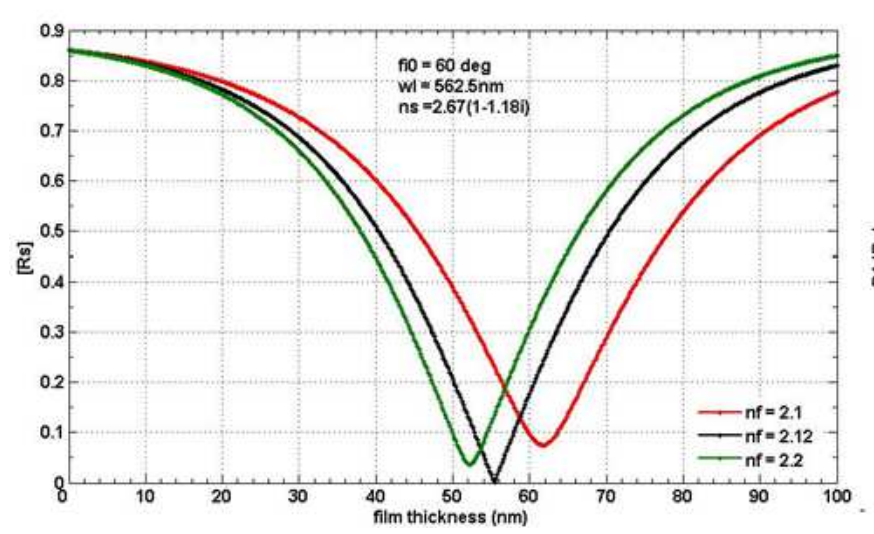

or

$$
\left|\widetilde{r}_{\mathrm{s}}^{12}+\widetilde{\mathrm{r}}_{\mathrm{s}}^{23} \cdot \exp \widetilde{\mathrm{D}}\right| \rightarrow 0
$$

The maximum of the curve $\Psi=\mathrm{f}\left(\mathrm{d}_{\mathrm{f}}\right)=90^{\circ}$ is obtained for $\mathrm{n}_{\Psi \max }=2.12$ and $\mathrm{d}_{\Psi_{\max }}=55.4 \mathrm{~nm}$.

Figure 5 presents the slopes of $\left|\widetilde{R}_{\mathrm{s}}\right|$ and its first derivative depending on the superficial film thickness.

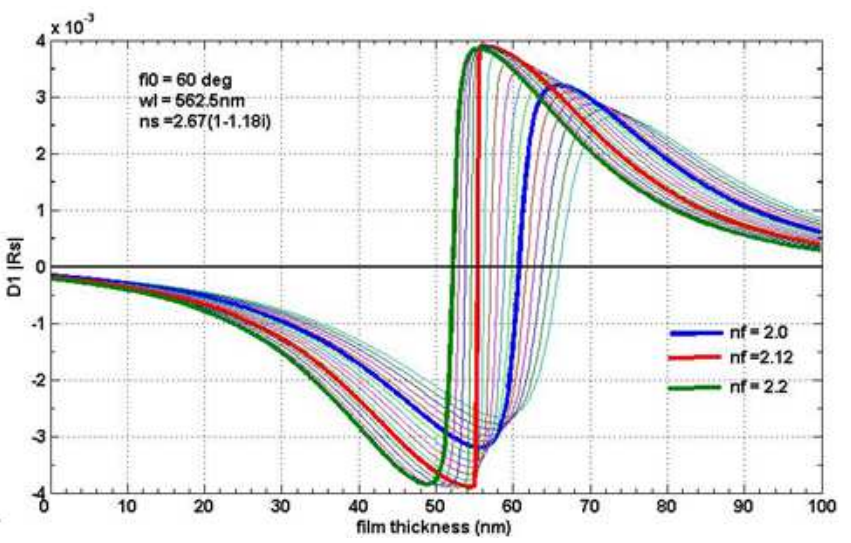

Figure 5. The curves $\left|\mathrm{R}_{\mathrm{s}}\right|=f\left(d_{f}\right)$ and $\frac{\partial}{\partial \mathrm{d}_{\mathrm{f}}}\left(\mid \widetilde{\mathrm{R}}_{\mathrm{s}}\right)=f\left(d_{f}\right)$ for superficial films, on a metallic substrate. $n_{\mathrm{s}}=2.67(1-1.18 i) ; \varphi_{0}=60^{\circ} ; \lambda=562.5 \mathrm{~nm}$.

At the point where $\Psi=90^{\circ},\left|\widetilde{R}_{\mathrm{s}}\right|=0$. The curve $\left|\widetilde{\mathrm{R}}_{\mathrm{s}}\right|=\mathrm{f}\left(\mathrm{d}_{\mathrm{f}}\right)$ suddenly changes its concavity and the first derivative of the modulus of complex parameter $\left|\widetilde{R}_{\mathrm{s}}\right|$ is indefinite. The ellipsometric parameter $\Delta$ is also indefinite, as shown in 
Figure $3 b$.

The curves $\left|\widetilde{R}_{\mathrm{s}}\right|=\mathrm{f}\left(\mathrm{d}_{\mathrm{f}}\right)$, Real $\left(\widetilde{\mathrm{R}}_{\mathrm{s}}\right)=\mathrm{f}\left(\mathrm{d}_{\mathrm{f}}\right)$ and Imag $\left(\widetilde{\mathrm{R}}_{\mathrm{s}}\right)$ are

presented in Figure 6.

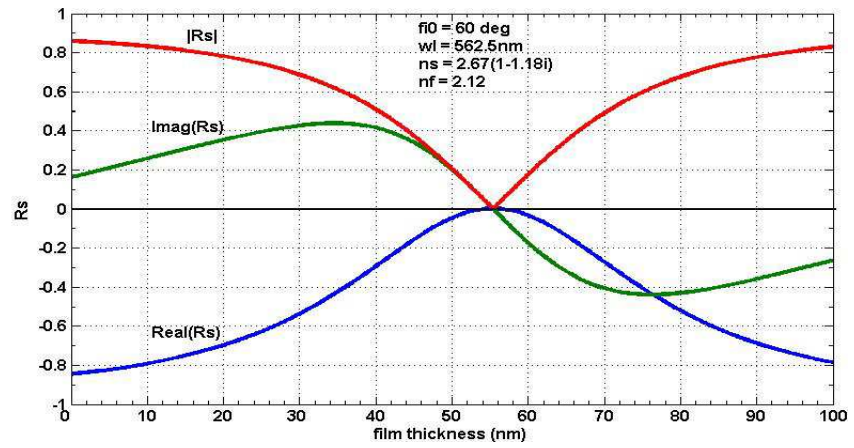

Figure 6. The curves $\left|\widetilde{\mathrm{R}}_{\mathrm{S}}\right|=f\left(d_{f}\right)$, Real $\left(\widetilde{\mathrm{R}}_{\mathrm{S}}\right)=f\left(d_{f}\right)$ and Imag $\left(\widetilde{\mathrm{R}}_{\mathrm{S}}\right)$ for superficial films, deposited on a metallic substrate. $n_{f}=2.12 ; n_{s}=2.67(1-1.18 i)$; $\varphi_{0}=60^{0} ; \lambda=562.5 \mathrm{~nm}$.

Information regarding the errors in the determination of the thickness and refractive index of a surface film is obtained by deriving the ellipsometric parameters $\Delta$ or $\Psi$ according to them.

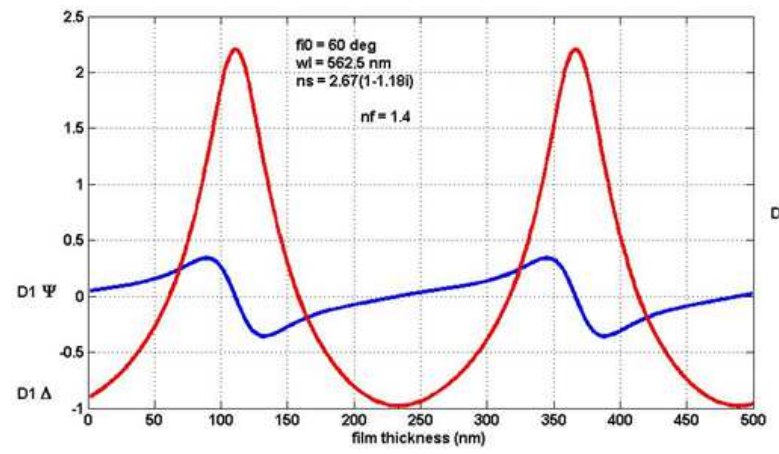

The first derivative of $\Delta$ and $\Psi$ depending on the superficial film thickness D1 $\Delta$ and D $1 \Psi$ are plotted in Figure 7 .

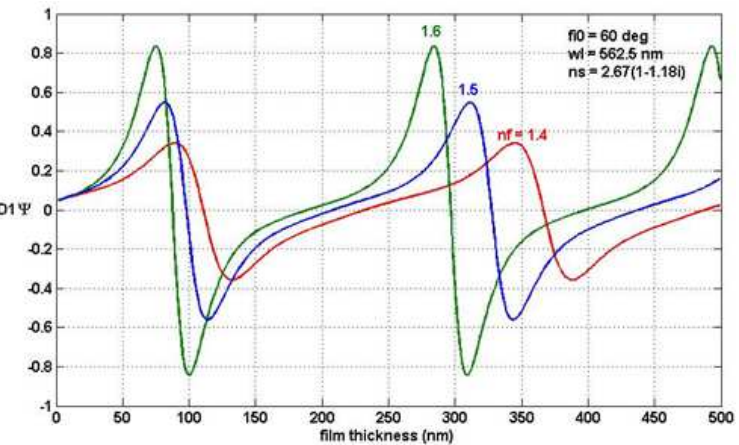

Figure 7. The first derivative of $\Delta$ and $\Psi$, depending on the superficial film thickness, deposited on a metallic substrate. $n_{s}=2.67(1-1.18 i) ; \varphi_{0}=60^{\circ} ; \lambda=562.5$ $n m$.

One can notice that $\mathrm{D}_{1} \Psi=0$ when the variation of $\Delta$ is maximum and $\mathrm{D}_{1} \Delta=0$ when the variation of $\Psi$ is maximum. Minimum errors in determining the refractive index $\mathrm{n}_{\mathrm{f}}$ and the thickness $d_{f}$ of superficial film are obtained when both ellipsometric parameters $\Delta$ and $\Psi$ shows large variations while the thickness or refractive index of the film is changing

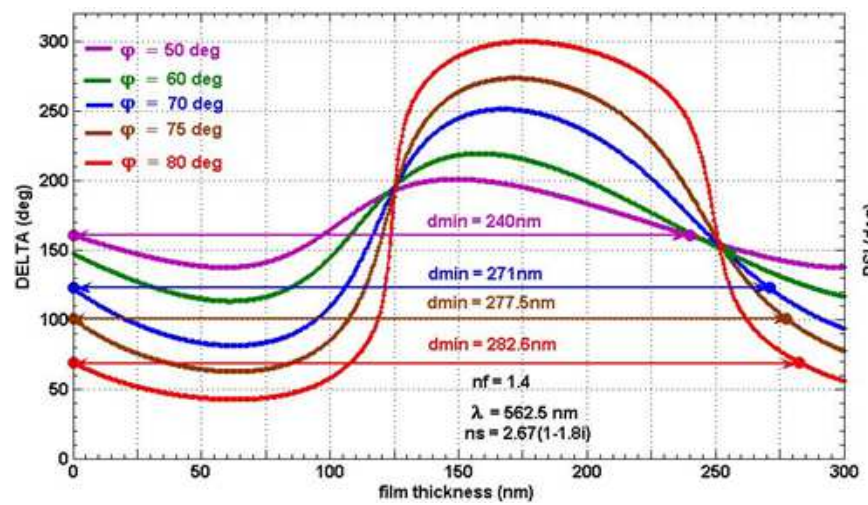

(the shaded area 1, in Figure 2a).

For superficial films with thicknesses close to $d_{\min }$ value, or multiple of this, the error in the determination of $n_{f}$ is very high (the shaded area 2 of Figure $2 a$ ).

\subsection{The Dependence of $d_{\min }$ on the Incidence Angle}

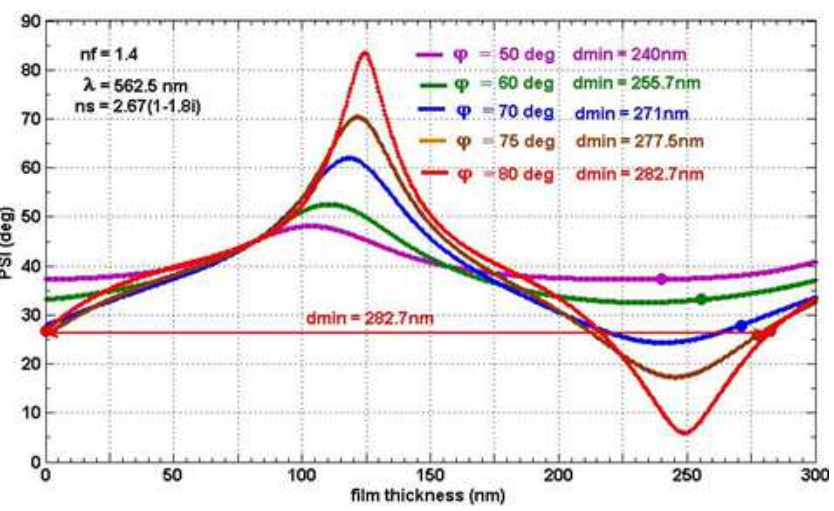

Figure 8. The curves $\Delta=f\left(d_{f}\right)$ and $\Psi=f\left(d_{f}\right)$ for superficial films, deposited on a metallic substrate, for different incidence angles. $n_{s}=2.67(1-1.18 i) ; \lambda=562.5$ $n m$.

The minimum thickness $d_{\min }$ after which $\Psi$ and $\Delta$ are repeated, increases when the angle of incidence $\varphi_{0}$ of the 
incident radiation increases, as shown in Figure 8, according to the relation (4).

An analysis of the dependence of the reflection coefficients $\widetilde{R}_{\mathrm{p}}$ and $\widetilde{\mathrm{R}}_{\mathrm{s}}$ on the incidence angle was performed by R.MA Azzam et al $[7,8,9]$.

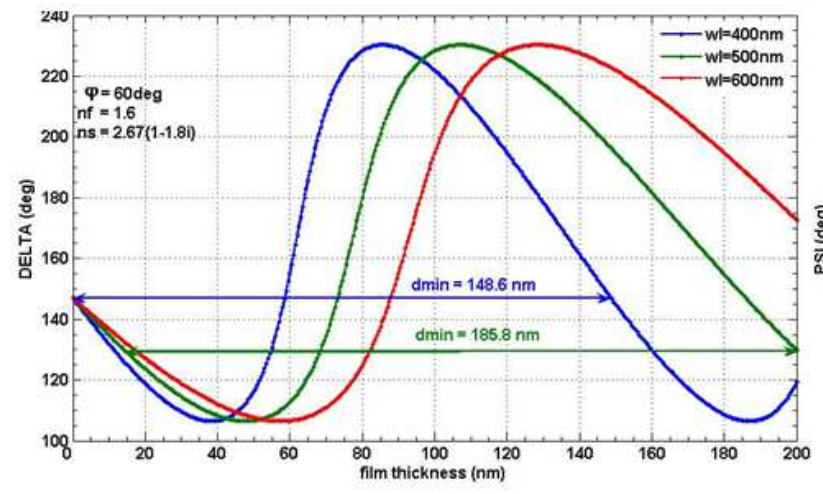

a)

\subsection{The Dependence of $d_{\min }$ on the Wavelength}

Thickness $d_{\min }$ increases when there are increases of wavelength $\lambda$, as shown in Figure 9, according to the relation (4).

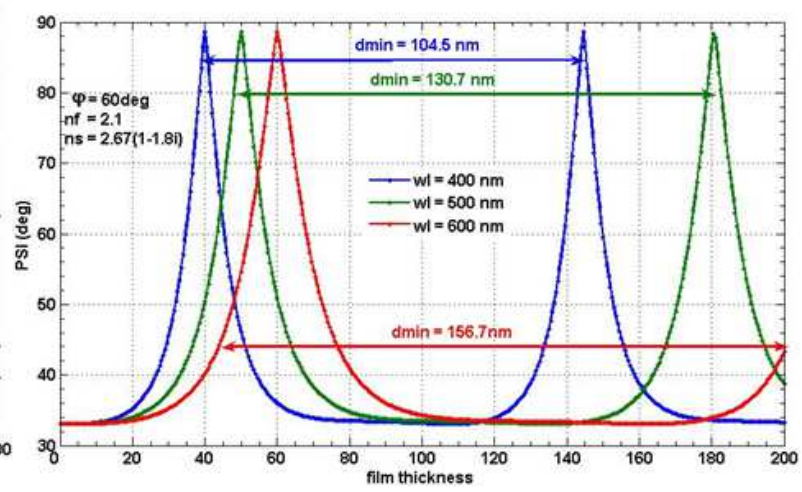

b)

Figure 9. The curves $\Delta=f(d f)$ and $\Psi=f(d f)$ for superficial films, deposited on a metallic substrate, at different wavelengts. $n_{s}=2.67(1-1.18 i)$; $\varphi_{0}=60^{0}$; a) $n_{f}=1.6$; b) $\left(\Psi=\Psi \max =90^{\circ}\right.$ for the same $n_{\Psi \max }=2.12$ irrespective of wavelength $\left.\lambda\right)$.

The analysis of these curves provides the following observations:

- The curves $\Delta=\mathrm{f}\left(\mathrm{d}_{\mathrm{f}}\right)$ and $\Psi=\mathrm{f}\left(\mathrm{d}_{\mathrm{f}}\right)$ have the same amplitude for a given value of the refractive index of the surface film.

- The film thickness $\mathrm{d}_{\Psi \max }$, for which $\Psi=90^{\circ}$, depends on the wavelength of the incident radiation. It increases when $\lambda$ increases. The ratio $d_{\Psi \max } / \lambda$ is constant. $d_{\Psi \max }=$ $\mathrm{k} \cdot \lambda$.

- The value of the refractive index $n_{\Psi \max }$ of the superficial film for which $\Psi=\Psi_{\max }=90^{\circ}$ does not depend on $\lambda$.

- When $\mid \tilde{\mathrm{\rho}} \rightarrow \infty$ or $\left|\widetilde{\mathrm{R}}_{\mathrm{s}}\right| \rightarrow 0$ the value of $\Psi_{\max }=90^{\circ}$ is obtained. It does not depend on the wavelength $\lambda$. The curves $\left|\widetilde{R}_{\mathrm{s}}\right|=\mathrm{f}\left(\mathrm{d}_{\mathrm{f}}\right)$ for superficial films with refractive index $n_{\Psi \max }$, at different wavelengths are presented in figure 10 .

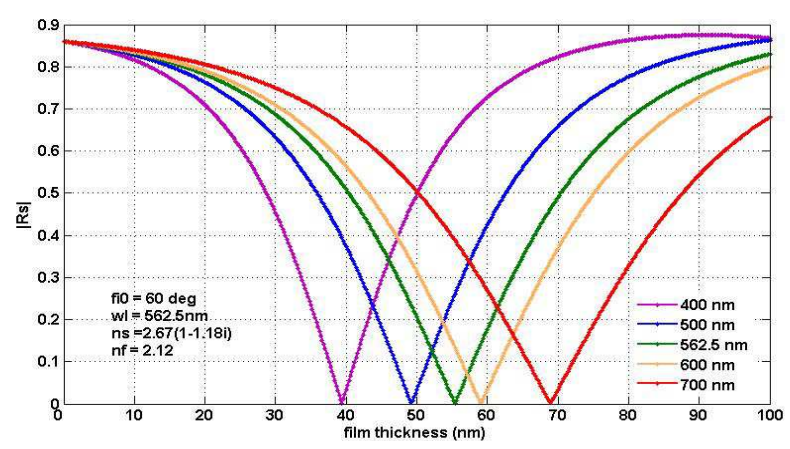

Figure 10. The curves $\left|\widetilde{\mathrm{R}}_{\mathrm{s}}\right|=f\left(d_{f}\right)$ for superficial films, on a metallic substrate, at different wavelengths; $n_{\Psi_{\max }}=2.12 ; n_{s}=2.67(1-1.18 i) ; \varphi=60^{\circ}$.

- For $\mathrm{n}_{\mathrm{f}}=\sqrt{1+\mathrm{n}_{0}^{2} \sin ^{2} \varphi_{0}} ; \mathrm{d}_{\min }=\lambda / 2$

- For non-absorbing superficial films with $\mathrm{n}_{\mathrm{f}}=1.3 \div 1.6$, always $d_{\min .}<\lambda$.

All computations were performed based on the Fortran program developed by McCrackin, amended as necessary [10]. The graphs were performed with the Matlab software.

\section{Conclusions}

Ellipsometrical analysis of external specular reflection of light on nonabsorbing superficial films allows us to know the factors that influence the ellipsometric measurement of the analyzed system. The right choice of the angle of incidence $\varphi_{0}$ and wavelength $\lambda$ depending on the refraction index of the superficial film, ellipsometrically measured, allows to be obtained minimal errors in the determination of its thickness and refraction index.

For optical nonabsorbing superficial films the curves $\Delta=\mathrm{f}\left(\mathrm{d}_{\mathrm{f}}\right)$ and $\Psi=\mathrm{f}\left(\mathrm{d}_{\mathrm{f}}\right)$ are periodical, while the curves $\Delta=\mathrm{f}(\Psi)$ are closed. The analysis of the periodicity of these curves allows us to correctly determine the film thickness for thicknesses greater than $d_{\text {min }}$. Knowing the minimal value of the film thickness from which the values of $\Delta$ and $\Psi$ are repeated eliminates possible errors in the determination of the superficial film thickness.

The value of $d_{\min }$ depends on the refractive index $n_{f}$ of the surface film, the incidence angle $\varphi_{0}$ and the wavelength $\lambda$ of the incident radiation.

The particular situation under analysis corresponds to a value of the ellipsometric parameter $\Psi=90^{\circ}$. Then $|\widetilde{\rho}| \rightarrow \infty$, or $\left|\widetilde{R}_{\mathrm{s}}\right| \rightarrow 0$. Useful applications of this interesting property are yet to be considered.

From the curve shape we can draw conclusions with respect to the domain of small errors, allowing us to correctly determine the thickness and refraction index of superficial films. 
Applications of these results are possible in the measurement and control of film thickness.

The results obtained testify that the materials with the refractive index close to $1.8 \div 2$ can be considered for photonic crystal application area.

\section{References}

[1] R.M.A. Azzam, N.M. Bashara, Ellipsometry and Polarized Light, North-Holland publ. Comp., Amsterdam - New York Oxford, 1977.

[2] S. Jitian, "The ellipsometrical study of adsorption-desorption of the corrosion inhibitors on metallic surfaces", Romanian Reports in Physics, Vol.65, No.1, pp. 204-212, 2013.

[3] S. Jitian, E. Chifu, "The Ellipsometric Study of Polimer Films on Metals", Studia Univ. Babeş-Bolyai. Chemia, Vol.31, No. 2, pp.69-75, 1986.

[4] H. Rosen, J. Shamir, "Interferometric determination of ellipsometric parameters" J. Phys. E: Sci. Instrum., Vol.11, No.9, pp.905-908, September 1978.

[5] K.H. Chen, C.C. Hsu, D.C. Su, "A method for measuring the complex refractive index and thickness of a thin metal film", Appl. Phys. B - Lasers and Optics, Vol.77, pp.839-842, December 2003.
[6] Juan E. González-Ramírez, Juan Fuentes, Luis C. Hernández, and Luís Hernández," Evaluation of the thickness in nanolayers using the Transfer Matrix method for modeling the spectral reflectivity", Research Letters in Physics, Vol. 2009, Article ID 594175, 2009.

[7] R. M.A. Azzam, M. Emdadur Rahman Khan, "Complex reflection coefficients for the parallel and perpendicular polarizations of a film-substrate system", Applied Optics, Vol.22, No.2, pp.253-264, January 1983.

[8] R. M.A. Azzam, T.F. Thonn, "Pseudo-Brewster and secondBrewster angles of an absorbing substrate coated by a transparent thin film", Applied Optics, Vol.22, No.24, pp.4155-4166, December 1983.

[9] R.M.A. Azzam, "Angle-of-incidence derivatives of the complex parallel and perpendicular reflection coefficients and their ratio for a film-substrate system "Opt. Acta, Vol.30, No.8, pp.1113-1124, August 1983.

[10] F.L. McCrackin, A Fortran Program for Analysis of Ellipsometer Measurement, Natl. Bur. Std., Technical Note 479, Washington, 1969. 